\title{
Chanwuyi Lifestyle Medicine Program Alleviates Immunological Deviation and Improves Behaviors in Autism
}

\author{
Agnes S. Chan ${ }^{1,2, *}$, Yvonne M. Y. Han ${ }^{3}$, Sophia L. Sze ${ }^{1,2}$, Chun-kwok Wong ${ }^{4}{ }^{\oplus}$, Ida M. T. Chu ${ }^{4}$ \\ and Mei-chun Cheung ${ }^{5}$ (D) \\ 1 Neuropsychology Laboratory, Department of Psychology, The Chinese University of Hong Kong, \\ Hong Kong, China; lmsze@cuhk.edu.hk \\ 2 Research Center for Neuropsychological Well-Being, The Chinese University of Hong Kong, \\ Hong Kong, China \\ 3 Department of Rehabilitation Sciences, The Hong Kong Polytechnic University, \\ Hong Kong, China; yvonne.han@polyu.edu.hk \\ 4 Department of Chemical Pathology, Prince of Wales Hospital, The Chinese University of Hong Kong, \\ Hong Kong, China; ck-wong@cuhk.edu.hk (C.-k.W.); idachu1219@gmail.com (I.M.T.C.) \\ 5 Department of Social Work, The Chinese University of Hong Kong, \\ Hong Kong, China; meichun@swk.cuhk.edu.hk \\ * Correspondence: aschan@psy.cuhk.edu.hk; Tel.: +852-394-366-54
}

Citation: Chan, A.S.; Han, Y.M.Y.; Sze, S.L.; Wong, C.-k.; Chu, I.M.T.; Cheung, M.-c. Chanwuyi Lifestyle Medicine Program Alleviates Immunological Deviation and Improves Behaviors in Autism. NeuroSci 2021, 2, 207-223. https:// doi.org/10.3390/neurosci2020015

Academic Editor: Asher Ornoy

Received: 8 April 2021

Accepted: 3 June 2021

Published: 7 June 2021

Publisher's Note: MDPI stays neutral with regard to jurisdictional claims in published maps and institutional affiliations.

Copyright: (c) 2021 by the authors. Licensee MDPI, Basel, Switzerland. This article is an open access article distributed under the terms and conditions of the Creative Commons Attribution (CC BY) license (https:/ / creativecommons.org/licenses/by/ $4.0 /)$.
Abstract: Given the association between deviated inflammatory chemokines, the pathogenesis of autism spectrum disorders (ASD), and our previous findings of the Chanwuyi Lifestyle Medicine Program regarding improved cognitive and behavioral problems in ASD, the present study aims to explore if this intervention can alter pro-inflammatory chemokines concentration. Thirty-two boys with ASD were assigned to the experimental group receiving the Chanwuyi Lifestyle Medicine Program for 7 months or the control group without a change in their lifestyle. The experimental group, but not the control group, demonstrated significantly reduced CCL2 and CXCL8, a trend of reduction in CCL5, and elevation of CXCL9. The experimental group also demonstrated significantly reduced social communication problems, repetitive/stereotypic behaviors, and hyperactive behaviors. The present findings support the potential efficacy and applicability of the Chanwuyi Lifestyle Medicine Program for reducing both behavioral problems and immunological dysfunction in ASD. Further studies are warranted to verify its treatment effect and its association with brain functions.

Keywords: autism spectrum disorders; intervention; chemokine; pro-inflammatory; behavioral problems

\section{Introduction}

Autism spectrum disorders (ASD) are neurodevelopmental disorders whose etiologies remain unknown, despite evidence that genetic, environmental, and immunological factors likely play important roles in its pathogenesis. As early as 40 years ago, a link between altered immune responses and ASD was first identified [1]. In recent decades, research on the immunological profile of ASD and identifying potential biomarkers of immunological dysfunction in ASD have become more vigorous [2]. Multiple studies have discovered that abnormal levels of various inflammatory cytokines/chemokines are found in the brain tissue, cerebrospinal fluid, plasma, and serum of individuals with ASD, which are believed to trigger neuroinflammation and disrupt neurodevelopment in ASD [3-7]. Cytokines are polypeptides with a major function of signaling immune responses to infection and inflammation. Chemokines, a form of cytokine, function as chemoattractant molecules to attract cells to migrate to local sites of infection and inflammation. In the central nervous system (CNS), chemokines regulate neuronal cell migration, proliferation, neuronal cell differentiation, and mediate communication between neurons and microglia.

Recent studies have repeatedly found elevated concentrations of CCL2, CCL5, and CXCL8 in brain tissues, cerebrospinal fluid, or peripheral blood samples in ASD as com- 
pared to their normal counterparts [3-7], which suggested a pathological link between the expressions of these chemokines and neuroinflammation in ASD. Relatively more empirical evidence revealed an association of the elevation in macrophage-related CCL2 and neutrophil-related CXCL8 with the impaired developmental and adaptive function associated with ASD as well as its core social communication and behavioral symptoms. As examples, higher CCL2 was related to greater problems in expressive language, fine motor skills, visual reception, and daily living skills [7]. In addition, higher CXCL8 was related to less social responsiveness, more stereotypic and hyperactive behaviors, and lower functional level in language and daily living skills in ASD [6]. Researchers even found that elevated CXCL8 increased the risk of having ASD [8] and postulated that CXCL8 is a correlator to the underlying nature of brain damage in ASD [9]. A few studies have examined the links between elevated T cells, eosinophils, and basophils-related CCL 5 and the risk of ASD and social deficits in ASD [3,7]. Our recent study [3] detected a significant correlation between CCL5 and inattention and hyperactive symptoms in ASD. Another chemokine, T helper type 1-related CXCL9, although relatively less investigated, is featured in emerging evidence suggesting that there is a deviated concentration in ASD. While some studies suggested the suppression of CXCL9 $[3,10]$, others reported an elevation in ASD [4].

Until now, there has been limited research on the effective intervention for tackling immunologic abnormalities as well as the symptomatology of ASD. Although some biological agents (e.g., transfer factor pentoxifyllinbasic fibroblast growth factor, intravenous immunoglobulin) have therapeutic effects on immunologic function and behavioral problems in ASD [11,12], this aspect of evidence warrants further verification in more well-designed controlled studies. On the other hand, an emerging line of research has proposed certain lifestyle factors (e.g., diet modification, exercise program) to improve the symptomatology of ASD, and some have reported positive outcomes [13-16] and improved immunologic condition in ASD $[17,18]$.

Lifestyle medicine, as defined by the American College of Lifestyle Medicine [19], is "the use of evidence-based lifestyle therapeutic approaches, such as a predominantly whole food, plant-based diet, regular physical activity, adequate sleep, stress management, avoidance of risky substance use, and other non-drug modalities, to prevent, treat, and reverse the lifestyle-related chronic disease". Cumulative scientific evidence supports the effects of replacing unhealthy lifestyles with positive ones for preventing, treating, and even reversing various common chronic diseases, including heart disease [20], cancer [21,22], stroke [23], diabetes [24], pain [25], and overweight/obesity [26]. Healthy lifestyles include eating whole, plant-based foods (i.e., veggies, fruits, beans, lentils, whole grains, nuts, and seeds) that are full of fiber, nutrients, and health-promoting; adopting a habit of regular moderate to vigorous intensity of physical activities; improving sleep quality through dietary and environmental changes; abstaining from tobacco; practicing effective coping and stress reduction techniques; and maintaining social connectedness to boost emotional resiliency and overall health. Findings from this line of research have demonstrated that comprehensive lifestyle changes can reverse the progression of even severe coronary heart disease, type 2 diabetes, prostate cancer, hypercholesterolemia, and high blood pressure [20,24,27].

While the majority of the empirical studies focused on the therapeutic benefits of lifestyle medicine on chronic illnesses, our research team has been exploring the effects of a traditional Chinese lifestyle intervention, the Chanwuyi Lifestyle Medicine Program [28,29], on improving various neurological conditions. The Chanwuyi Lifestyle Medicine Program is a comprehensive lifestyle intervention approach and is developed upon the medical principles originating from the Chinese Chan Medicine. Chinese Chan Medicine is a holistic approach facilitating better well-being and quality of life through modification of one's life habits, including diet modification, mind-body exercises, and self-realization. Cumulative empirical and clinical evidence of this Chinese lifestyle medicine program have suggested positive treatment outcomes on brain functions, mood state, problematic behaviors, and the physical health of normal individuals [30-32], elderly with memory complaints [33-35], 
and patients with various brain disorders, including ASD [36-41], depression [42-44], and epilepsy [45]. Children with ASD, who have adopted our Chan-based diet for 1 month, showed significantly enhanced executive functions (including cognitive flexibility, impulse control, and planning ability) and reduced social communication problem and repetitive behaviors, whereas those without changes in their diet did not show such improvement [38]. Those positive changes in cognitive performance and problem behaviors were in line with their increased EEG brain activity at the anterior cingulate cortex. Another two randomized controlled studies have detected a positive effect for a 1-month regular practice of the mindbody exercises (a component in our lifestyle intervention) on impulse control ability [39] and memory retrieval strategy [41] in children with ASD, together with their elevated neurophysiological activity in anterior cingulate cortex and fronto-posterior EEG coherence, respectively. Such beneficial effects were not observed in the control group practicing progressive muscle relaxation. Two other case studies on ASD with the adoption of the Chanwuyi Lifestyle Medicine Program also demonstrated significant improvement in executive functions, memory, and symptomatology of ASD [36,37].

Nevertheless, in spite of the promising results of the lifestyle program on ASD, these studies have been relatively short-term, with the intervention lasting just 1 month. Therefore, the present study aims to explore its treatment effect over a longer term (i.e., a 7-month Chanwuyi Lifestyle Medicine Program). Our previous studies are primarily focusing on the treatment effects on autistic symptoms, cognitive function, and brain physiological state; we have not yet explored its possible effects on the immune function of children with ASD. Previous empirical evidence reported enhanced cognitive functions and improved problem behaviors, and years of clinical observation also found that children adopting the lifestyle changes showed improved gastrointestinal function and sleep quality and reduced frequency of sickness and nasal/skin allergy. The mechanism underlying those positive changes might have an immunological basis. Therefore, it is anticipated that children receiving the 7-month Chanwuyi Lifestyle Medicine Program will demonstrate a positive change in their inflammatory chemokine profile. As reported in our recent study on the distinctive chemokine profile in ASD [3], children with ASD demonstrated elevated CCL2, CCL5, and CXCL8 and reduced CXCL9. Therefore, we anticipate that after the intervention, there will be a decrease in CCL2, CCL5, CXCL8, and an increase in CXCL9 in the experimental group. Such immunologic alteration would also be in line with their improvement in social and behavioral problems. Such positive changes are not expected for children in the control group.

\section{Materials and Methods}

\subsection{Participants}

Thirty-two boys aged 6 to 18 years participated voluntarily in the present study. Written informed consents were obtained from their parents before the study. All children were diagnosed by a clinical psychologist based on the diagnostic criteria of Diagnostic and Statistical Manual of Mental Disorders, 5th edition (DSM-5) [46], and the caregivers' responses were obtained from the Autism Diagnostic Interview-Revised (ADI-R) [47]. Children who had any neurological disorder or infectious and allergic diseases such as influenza, allergic asthma, and atopic dermatitis, or were prescribed immunosuppressive drugs were excluded from the study. All children were not taking any psychotropic medication, except for one child who was prescribed with Risperdal. Among the 32 boys, 20 of them were trained with the Chanwuyi Lifestyle Medicine Program (i.e., the experimental group), whereas the remaining 12 boys who did not receive the lifestyle medicine program served as the control group.

Table 1 presents the demographic and clinical characteristics of the two groups of children. These groups were matched for age $(t=-1.71, p=0.10)$ and level of intelligence $(t=1.46, p=0.15)$, as estimated by the short form of the Chinese version of the Wechsler Intelligence Scale for Children, 4th edition (Hong Kong) (WISC-IV(HK) [48]. These children also showed a comparable level of autistic symptoms as assessed by the ADI-R $(t=-1.27$ 
to $0.11, p=0.22$ to 0.91 ), and social and behavioral problems, as measured by the Social Responsiveness Scale, 2nd edition (SRS-2) [49] $(t=-0.80, p=0.43)$, the Hyperactivity subscale of short version of Conners' Rating Scales-Revised (CRS-R) [50] ( $t=2.00, p=0.06)$, and the Stereotypic Behavior and Hyperactive/Noncompliance subscales of Aberrant Behavioral Checklist (ABC) [51] $(t=0.64$ and 1.72, $p=0.53$ and 0.10).

Table 1. Demographic and clinical characteristics of children with ASD in the experimental and control group at the baseline.

\begin{tabular}{|c|c|c|c|c|}
\hline & Experimental Group $(n=20)$ & Control Group $(n=12)$ & $t$ & $p$ \\
\hline Age, years & $11.08(3.54)$ & $13.39(3.97)$ & -1.71 & 0.10 \\
\hline IQ & $86.65(16.55)$ & $80.00(9.14)$ & 1.46 & 0.15 \\
\hline ADI-R Social Interaction & $19.70(5.54)$ & $20.17(5.80)$ & -0.23 & 0.82 \\
\hline ADI-R Communication & $14.35(5.64)$ & $16.67(3.68)$ & -1.27 & 0.22 \\
\hline ADI-R Stereotyped Behavior & $5.25(2.29)$ & $5.17(1.70)$ & 0.11 & 0.91 \\
\hline SRS-2 Total Score & $80.45(26.29)$ & $87.42(19.09)$ & -0.80 & 0.43 \\
\hline CRS-R Hyperactivity & $6.40(4.76)$ & $3.33(3.03)$ & 2.00 & 0.06 \\
\hline ABC Stereotypic Behavior & $4.45(3.95)$ & $3.58(3.18)$ & 0.64 & 0.53 \\
\hline ABC Hyperactivity/Noncompliance & $19.00(10.31)$ & $13.17(7.25)$ & 1.72 & 0.10 \\
\hline
\end{tabular}

Notes: Data are presented as means (SD). Abbreviations: ABC—Aberrant Behavior Checklist; ADI-R-Autism Diagnostic InterviewRevised; ASD—Autism Spectrum Disorder; CRS-R—The short form of Conners' Rating Scales—Revised; IQ—Intelligence Quotient as assessed by the short form of Chinese version of Wechsler Intelligence Scale for Children, 4th edition; SRS-2-Social Responsiveness Scale, 2nd edition.

\subsection{Procedures}

This clinical study was conducted in accordance with the World Medical Association Declaration of Helsinki. The research protocol was approved by the Ethical Review of Research Project Involving Human Subjects at the Hong Kong Polytechnic University (Reference No.: HSEARS20111215002). We recruited the participants through advertisement in mass media and the email sent to our laboratory database. Children who had previously been diagnosed to have ASD or a tendency of ASD and who did not have any neurological disorder or were not prescribed immunosuppressive drugs were eligible to participate in the study. Figure 1 illustrates the flow of the experimental procedure of the present study.

After briefing the parents of all children about the procedure of the experiment and obtaining their informed consent, peripheral blood samples of each child were collected at a medical clinic by a registered nurse using venipuncture. The collected blood samples, $3 \mathrm{~mL}$ of EDTA blood and $3 \mathrm{~mL}$ of clotted blood, were centrifuged at $3000 \mathrm{rpm}$ for $15 \mathrm{~min}$, and then, the harvested serum was stored at $-80{ }^{\circ} \mathrm{C}$ in a clinical laboratory until chemokine levels were measured. The processing and analysis of blood samples were performed by an experienced laboratory technician who was blind to our experimental design and group assignment. The serum chemokine concentrations were measured according to the manufacturer's instructions.

On a separate day, the parent of each child was invited to a clinical interview conducted by a clinical psychologist and a well-trained research assistant. During the interview, parents were asked about the developmental and medical history of their children, and their social and behavioral characteristics based on a structured interview and standardized questionnaires. Based on the DSM- 5 criteria and the information collected in the ADI-R, the clinical psychologist re-confirmed the diagnosis of each child. The research assistant guided parents to complete the standardized questionnaires about their child's everyday socialization and behavior repertoire. The clinical psychologist and research assistant were blinded to the rationale of the study and the group assignment.

Once the above baseline measures were collected, the participants were assigned into the experimental or the control group based on convenience sampling. Children who were available and willing to receive the lifestyle intervention were allocated to the experimental group, whereas those who were not willing or not available to join the intervention program were allocated to the control group. Children of the experimental group were arranged for a 7-month Chanwuyi Lifestyle Medicine Program, whereas children in the control 
group were not. The intervention was conducted at the Chinese University of Hong Kong, once per week, for 7 months on a group basis. Each training session lasts for $1.5 \mathrm{~h}$. The experimental group was divided into two groups based on their level of education (primary and secondary level). The two groups were run consecutively by well-trained trainers based on a standardized intervention protocol. The progress and quality of training were closely monitored by the first author, who has developed the intervention protocol, through weekly clinical meeting and regular on-site supervision throughout the intervention period.

After the intervention, the same procedure of blood samples collection and assay for the children as well as assessing the social and behavioral problems using the standardized questionnaires with the parents were conducted for both the experimental and control groups. These data served as the outcome measures at post-intervention to evaluate the treatment effect.

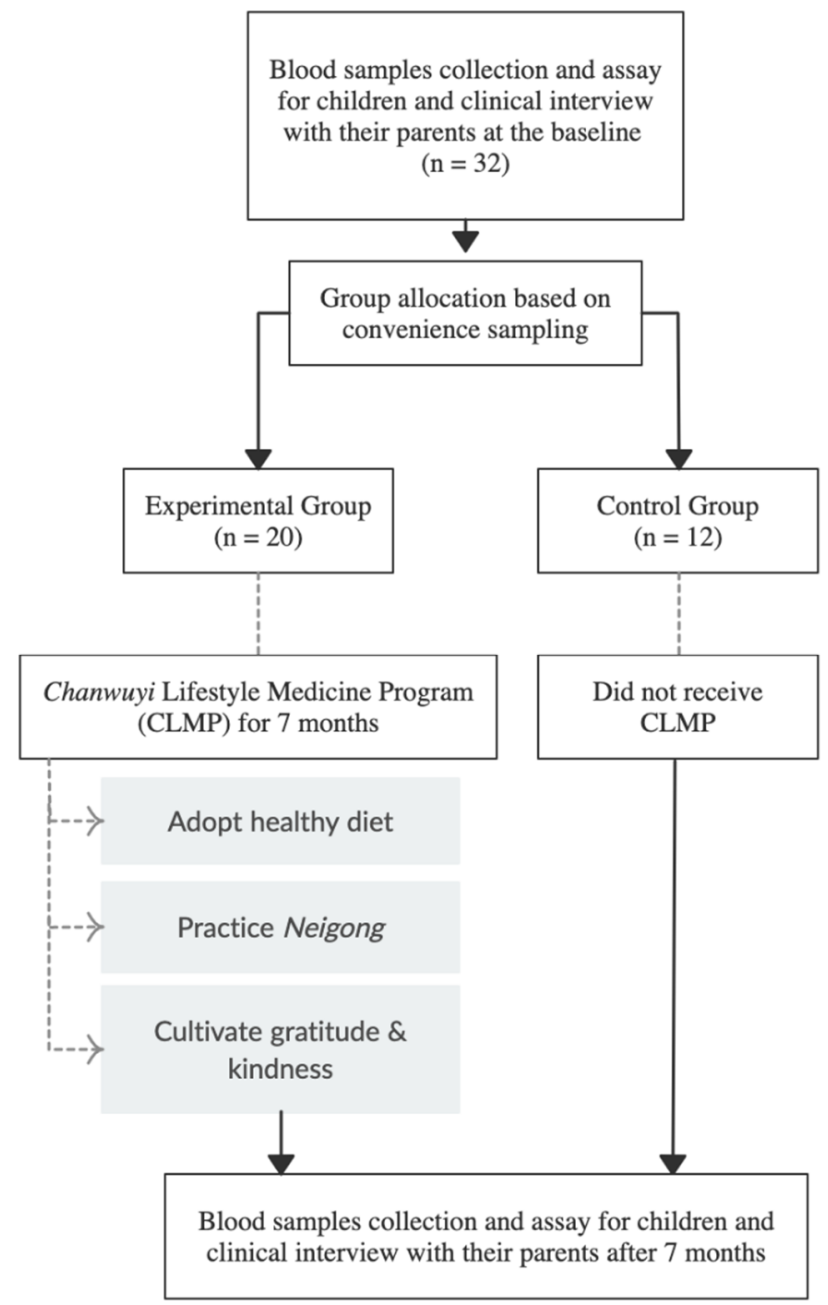

Figure 1. Flow of experimental procedure.

\subsection{Intervention}

The Chanwuyi Lifestyle Medicine Program was developed upon a traditional Chinese health-promoting lifestyle and Chan-based culture, namely Chanwuyi (i.e., Chan, martial arts and healing). Chanwuyi is a well-developed form of lifestyle medicine that is based on its Chan medical principles. According to the principles, our living style and attitude are the key factors for our health status. This intervention emphasizes on adopting healthy dietary, exercise habits, and positive living attitude to maintain the health of the mind and the body. Given that the focus of this intervention is on modifying the daily living habit of the children, they were allowed to continue with their other existing treatments, if any. This 
lifestyle medicine program has been empirically studied since 2007 and clinically adopted since then.

Throughout the 7-month intervention, children and their parents were taught the fundament principles and techniques of Chanwuyi Lifestyle Medicine Program. Each training session was divided into three subsections. (1) Advise adoption of a plant-based diet and avoid intake of food that generates excessive internal heat and adversely affects moods and physical health according to the Chan medical principle. It is recommended to eat four major categories of healthy foods: (a) fresh fruits and (b) vegetables in different colors and eat the whole plant; (c) plant-based protein (seeds, beans, nuts), and (d) whole grains (e.g., barley, millet, brown rice). Each category of foods accounts for a quarter portion of the entire meal to ensure balanced nutrition consumed. By upholding the natural and relaxing principle, participants drink when feeling thirsty, eat until feeling sufficient with joyful and relaxed attitude. Food-to-avoid include ginger, garlic, green onions, spicy foods, eggs, meat, fish, junk food, cold drink and food, and food with trans-fat and added sugar. Children were encouraged to change their diet at their own pace. If for whatever reason they could not fully adopt the plant-based diet, they were recommended to reduce intake of the food that is not recommended and increase the proportion of fruits and vegetables. Such a plant-based diet has been empirically studied to be effective in reducing behavioral problems and modulating abnormal brain activity in children with ASD [38]; (2) Practice a form of mind-body exercise, namely Neigong, which composes sets of breathing exercises and gentle and calm movement. This type of exercise, similar to Tai Chi, is classified as a moderate intensity level of physical activity. For detail of instruction please visit the website https:/ / chanwuyilifestyle.psy.cuhk.edu.hk (accessed on 11 February 2021). Our previous research has found that regular practice of Neigong helps to induce a relaxed and attentive brain state [31] and to improve impulse control and memory ability of children with ASD [39,41]. Children were encouraged to practice Neigong daily at home. (3) Selfrealization is a process of self-awareness and understanding of how our greed, anger, and obsession negatively affect our behaviors, emotions, and health. During the program, children learned to cultivate gratitude and kindness to get rid of those negative beliefs and to combat stress through interactive games and self-expression.

Each child was asked to complete the log record of home practice. The log record covered aspects of diet modification, exercise practice, and attitude cultivation in everyday living. Their records were reviewed weekly. Children who had made an effort to change their living habits and attitudes were reinforced with praise and stickers as encouragement.

\subsection{Immunological Measures}

The concentrations of chemokines CCL2, CCL5, CXCL8, and CXCL9 were measured using the $\mathrm{BD}^{\mathrm{TM}}$ human chemokine cytometric bead array (CBA) reagent (Becton Dickinson Biosciences Pharmingen, San Jose, CA, USA). The multi-fluorescence BD FACSCalibur ${ }^{\mathrm{TM}}$ flow cytometer identifies and enumerates chemokine subsets using BD CellQuest ${ }^{\mathrm{TM}}$ and $\mathrm{BD}^{\mathrm{TM}} \mathrm{CBA}$ software.

\subsection{Behavioral Measures}

\subsubsection{Social Communication and Stereotypic/Repetitive Behaviors}

Socialization deficits and stereotypic or repetitive behaviors are two core symptoms of ASD, which are tapped by the total score of the SRS-2 and the Stereotypy subscale of ABC. The SRS-2 is a sensitive standardized parent-report questionnaire that measures the aspects of social awareness, social cognition, social communication, social motivation, and restricted interests and repetitive behavior in ASD. It comprises 65 items scored on a 0 to 3 scale, where its maximum score is 195. A higher score indicates more severe problems. The Stereotypy subscale of $A B C$ comprises seven items scored on a 0 to 3 scale with behaviors scoring a 3 being most problematic. The maximum score is 21 . It provides a measure of stereotypic behaviors in terms of repetitive motor movements. 


\subsubsection{Hyperactivity Behaviors}

Hyperactive behaviors were measured by the Hyperactivity subscales of the short version of CRS-R and Hyperactivity/Noncompliance subscale of ABC. The Hyperactivity subscales comprises six items scored on a 0 to 3 scale, where its maximum score is 18 . The Hyperactivity/Noncompliance subscale of $\mathrm{ABC}$ comprises 16 items rated on a 4-point scale, where its maximum score is 48 . For both measures, a higher score indicates the higher severity of the problem.

\subsection{Data Analyses}

Between-group comparison on demographic details and baseline measures was performed by independent sample $t$-tests. The comparison between pre- and post-training measures of the two groups was performed by repeated measures analysis of variance (ANOVA) and followed by paired samples $t$-tests to delineate the direction and extent of changes within each group of children. To avoid inflation of Type I error resulting from multiple comparisons, the alpha value was adjusted by dividing the number of comparisons within the same construct (i.e., 0.013). The effect size was also computed to indicate the degree of between-group difference across time.

\section{Results}

\subsection{Chanwuyi Lifestyle Medicine Program Modulated Pro-Inflammatory Chemokines in ASD}

At the baseline, the experimental group demonstrated a similar profile of chemokine concentrations to that of the control group (Table 2). There was no significant difference between the two groups in the four immunological measures given the adjusted alpha value at $0.013, t$ ranges from 0.66 to $2.15, p \geq 0.04$.

Table 2. Comparison of chemokine concentrations between experimental and control group at the baseline.

\begin{tabular}{ccccc}
\hline & $\begin{array}{c}\text { Experimental Group } \\
(\boldsymbol{n}=\mathbf{2 0})\end{array}$ & Control Group $(\boldsymbol{n = 1 2 )}$ & $\boldsymbol{t}$ & $\boldsymbol{p}$ \\
\hline CCL2 & $193.73(60.25)$ & $156.03(38.83)$ & 2.15 & 0.04 \\
CCL5 & $43,176.60(18,019.80)$ & $37,264.78(8839.55)$ & 1.24 & 0.23 \\
CXCL8 & $86.81(27.87)$ & $80.20(26.61)$ & 0.66 & 0.51 \\
CXCL9 & $232.33(96.57)$ & $347.52(257.99)$ & 1.49 & 0.16 \\
\hline
\end{tabular}

Notes: Data are presented as means (SD).

Separate repeated measures ANOVA was performed to examine the changes in each chemokine concentration between two groups across two measurement time points. Figure 2 shows the pre-post changes of each chemokine concentration in the two groups. Statistical results showed a significant Group (Experimental vs. Control) $\times$ Time (Pre vs. Post) interaction effect on the concentration level of CCL2 and CXCL8 ( $F=4.38$ and 10.98, $p=0.045$ and 0.002), partial eta squared $=0.13$ (medium effect size) and 0.27 (large effect size), respectively. Post hoc paired sample $t$-tests found that the experimental group demonstrated a significant reduction in the concentration level of CCL2 and CXCL8 after a 7-month intervention $(t=4.07$ and 4.71 , effect size $=0.91$ and 1.05 respectively, $p \leq 0.001)$. In contrast, the concentration level of CCL2 and CXCL8 remains constant after 7 months in the control group $(t=1.48$ and $-1.10, p=0.17$ and 0.29 , effect size $=0.43$ and 0.32 respectively).

The result of repeated measures ANOVA for CCL5 demonstrated a significant Time effect $(F=11.65, p=0.002$, partial eta squared $=0.28$ (large effect size)), but not Group $\times$ Time interaction effect $(F=0.63, p=0.43$, partial eta squared $=0.02$ (small effect size)). A post hoc paired samples $t$-test found a significant reduction in CCL5 concentration in the control group $(t=3.62, p=0.004$, effect size $=1.05)$ and a trend of reduction in the experimental group $(t=1.90, p=0.07$, effect size $=0.42)$. It should be noted that the SDs of the experimental group (pre: 18,019.79; post: 16,857.66) before and after intervention are more than double those of the control group (pre: 8839.55; post: 6950.12). Given that the effect size of the pre-post difference in the experimental group is approaching the medium level, 
therefore, the non-significant change in the experimental group is probably due to the large variability within the group. Furthermore, the result of the independent samples $t$-test showed that the pre-post difference (reduced by 6100.13 in the experimental group and 9804.23 in the control group) in CCL5 concentration between the two groups is not significant $(t=0.80, p=0.43)$.

a)

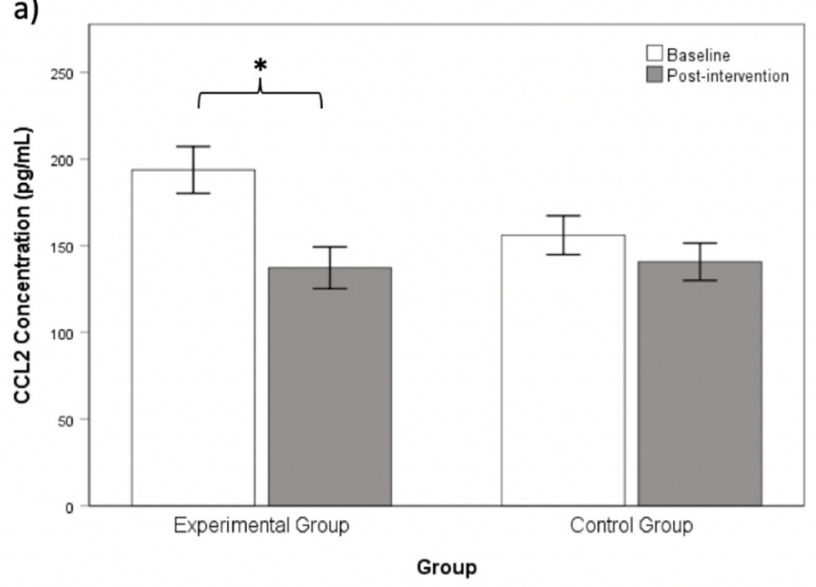

b)

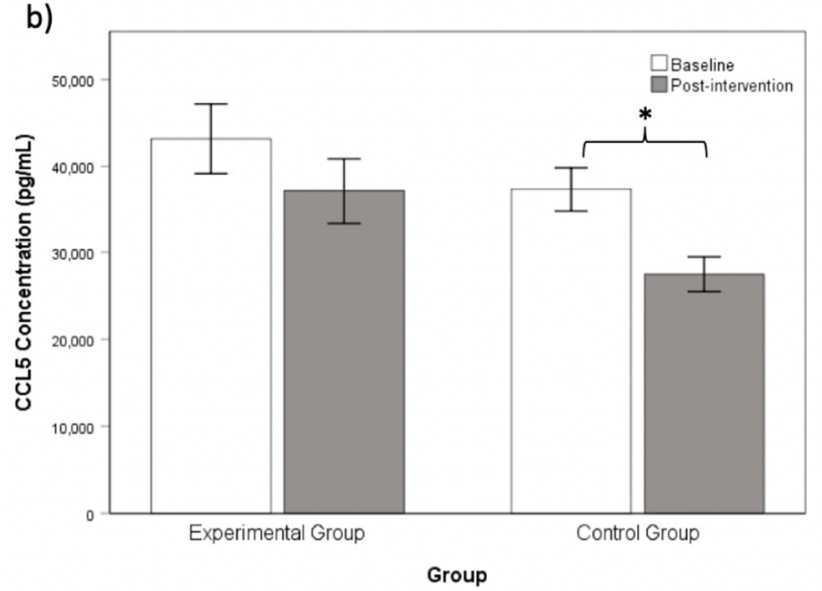

c)

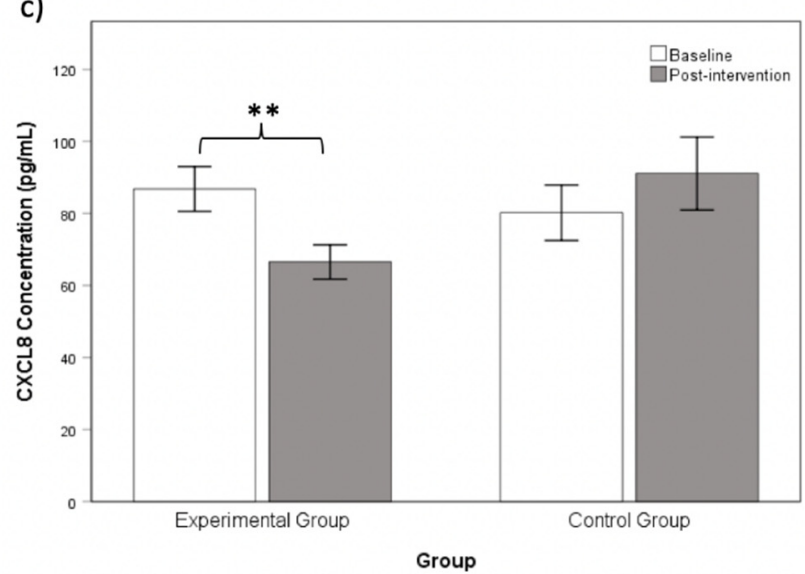

d)

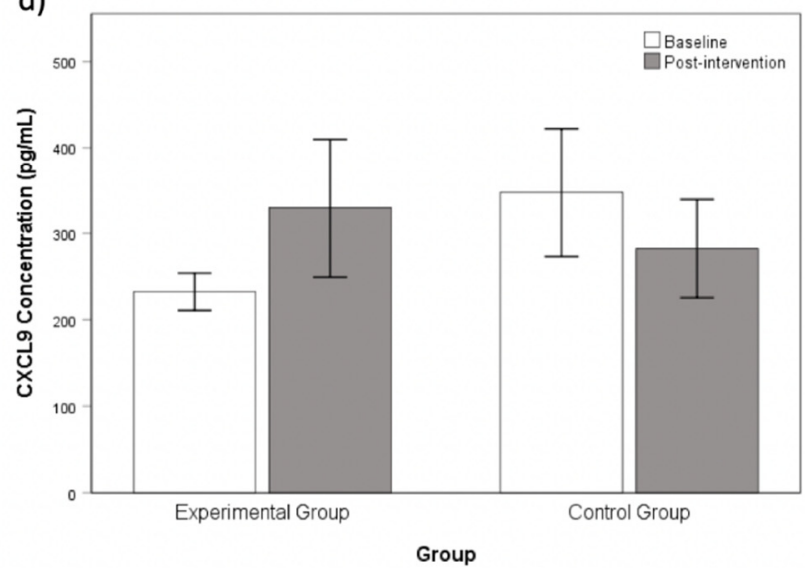

Figure 2. Mean concentrations of four chemokines in the experimental and control group before and after intervention. The experimental group shows a significant reduction in (a) CCL2 and (c) CXCL8 and a trend of reducing (b) CCL5 and (d) CXCL9, whereas the control group shows a significant reduction in (c) CCL5. ${ }^{*} p<0.013,{ }^{* *} p \leq 0.001$ in paired samples $t$-test. Error bars represent 1 standard error \pm mean.

Although the Group $\times$ Time interaction effect on CXCL9 was not significant in repeated measures ANOVA $(F=2.11, p=0.16$ ), its effect size reached medium level (partial eta squared $=0.07$ ), and eyeball inspection of the direction of alteration in CXCL9 concentration suggested a sensible and noteworthy trend of change in two groups. As reported in our previous study [3], children with ASD were found to have an approximately two-fold lower level of CXCL9 concentration than typically developed children, and this betweengroup difference was statistically significant. Their reduced CXCL9 concentration was also found to be associated with higher social and behavioral problems. In the present study, children from the experimental group showed a $42 \%$ elevation in CXCL9 concentration after intervention (from 232.33 to 329.35 ), whereas the control group showed a $19 \%$ reduction in CXCL9 concentration after 7 months (from 347.52 to 282.17). In addition, the variability in the experimental group at post-intervention $(\mathrm{SD}=358.04)$ is much larger than that of the control group ( $\mathrm{SD}=196.51)$. Although the pre-post changes in the experimental and control groups did not reach statistical significance $(t=-1.29$ and $0.93, p=0.21$ and 0.37 , effect size $=0.29$ and 0.27 respectively), the incremental trend in CXCL9 concentration 
mainly found in the experimental group might suggest a potential positive effect of Chanwuyi Lifestyle Medicine Program on this chemokine subset that would be worth further investigation as part of a future study with a larger sample size.

\subsection{Chanwuyi Lifestyle Medicine Program Reduced Core Symptoms in ASD}

The positive effect of the Chanwuyi Lifestyle Medicine Program was also observed in reducing the severity level of core autistic symptoms. The SRS-2 total score and Stereotypy subscale score in $\mathrm{ABC}$ was adopted to evaluate the social communication ability and stereotypic/repetitive behaviors of the children. Repeated measures ANOVA demonstrated significant Time effect on both measures $(F=10.85$ in SRS-2 and 6.83 in ABC, $p=0.003$ and 0.014 respectively). Yet, there was no significant interaction effect identified ( $F=0.53$ and $0.03, p=0.47$ and 0.87 respectively). Figures 3 and 4 demonstrate the change in mean rating on the respective scales before and after the intervention. A post hoc paired samples $t$-test revealed that the experimental group showed a significant reduction in core autistic symptoms, as measured by the SRS-2 after the intervention $(t=2.89, p=0.009$, effect size $=0.65)$ (Figure 3). Although children from the control group demonstrated a trend of reduction in the SRS-2 total score $(t=2.30, p=0.042$, effect size $=0.66)$, such change did not reach statistical significance when the adjusted alpha level (0.013) was adopted. Interestingly, the pre-post change in SRS-2 total score was significantly correlated with the change in CCL5 concentration $(r=0.41, p=0.02)$; that is, the greater the extent of reduction in CCL5 concentration, the larger the reduction in SRS-2 score (i.e., fewer social communication problems). Yet, any change in SRS-2 total score did not correlate with other chemokine indices ( $r$ ranges from -0.01 to $0.23, p$ ranges from 0.21 to 0.96 ). For the Stereotypy subscale in $\mathrm{ABC}$, both groups showed reduced scores, but the change was not statistically significant at an adjusted alpha level $(t=2.15, p=0.045$, effect size $=0.48$ in the experimental group, and $t=1.73, p=0.11$, effect size $=0.50$ in the control group) (Figure 4). There was no significant correlation between any chemokine concentration levels and the Stereotypy score ( $r$ ranges from -0.06 to $-0.17, p$ ranges from 0.35 to 0.76 ).

Since the total score of SRS-2 comprises the Social Communication Index (SCI) and Restricted and Repetitive Behavior Index (RRBI), further analysis was performed to delineate whether the reduction in total score was due to a reduction in either one or both of the index scores. The results of a paired samples $t$-test revealed that the experimental group showed a significant reduction in both SCI and RRBI $(t=2.75$ and 2.80 respectively, $p \leq 0.013)$, whereas the control group did not $(t=2.25$ and 2.04 respectively, $p \geq 0.05)$. The pre- and post-mean (SD) scores of SCI in the experimental group were 66.75 (21.05) and 54.15 (17.04), and those in the control group were 73.67 (17.16) and 65.00 (22.05). The pre- and post-mean (SD) scores of RRBI in the experimental group were 13.70 (6.55) and 9.20 (5.09), and those in the control group were 13.75 (3.89) and 11.50 (4.70). Therefore, this suggests that the Chanwuyi Lifestyle Medicine Program significantly reduces the two core clinical symptoms of ASD in the experimental group. The SCI score was positively correlated with CCL5 concentration $(r=0.48, p=0.005)$ but not with other chemokine indices ( $r$ ranges from 0.02 to $0.23, p$ ranges from 0.21 to 0.93 ). There was no significant correlation between any chemokine indices and RRBI score ( $r$ ranges from -0.08 to $-0.25, p$ ranges from 0.16 to 0.68$)$.

\subsection{Chanwuyi Lifestyle Medicine Program Reduced Hyperactivity Behaviors in ASD}

In addition to core autistic symptoms, children who had received the Chanwuyi Lifestyle Medicine Program showed a significant reduction in hyperactive behaviors as measured by the Hyperactivity subscale in CRS-R and Hyperactivity/Noncompliance subscale in ABC. Figure 5 shows the pre-post changes in measures of hyperactive behaviors in both groups. In repeated measures ANOVA, there is a significant Group $\times$ Time interaction effect on CRS-R subscale $(F=7.75, p=0.009$, partial eta squared $=0.21$ (large) $)$, and a significant Time effect on the $\mathrm{ABC}$ subscale $(F=1.99, p=0.17$, partial eta squared $=0.06$ (medium)). A post-hoc paired samples $t$-test showed that the experimental group 
demonstrated significantly reduced hyperactivity behavior in both CRS-R and ABC subscales $(t=3.65$ and 3.15, $p=0.002$ and 0.005 , effect size $=0.82$ and 0.70 , respectively). In contrast, the control group did not show such a significant reduction $(t=-0.50$ and 1.38, $p=0.63$ and 0.20 , effect size $=0.14$ and 0.40 , respectively). The two hyperactivity measures were not correlated with any chemokine indices ( $r$ ranges from 0.03 to $0.22, p$ ranges from 0.22 to 0.86$)$.

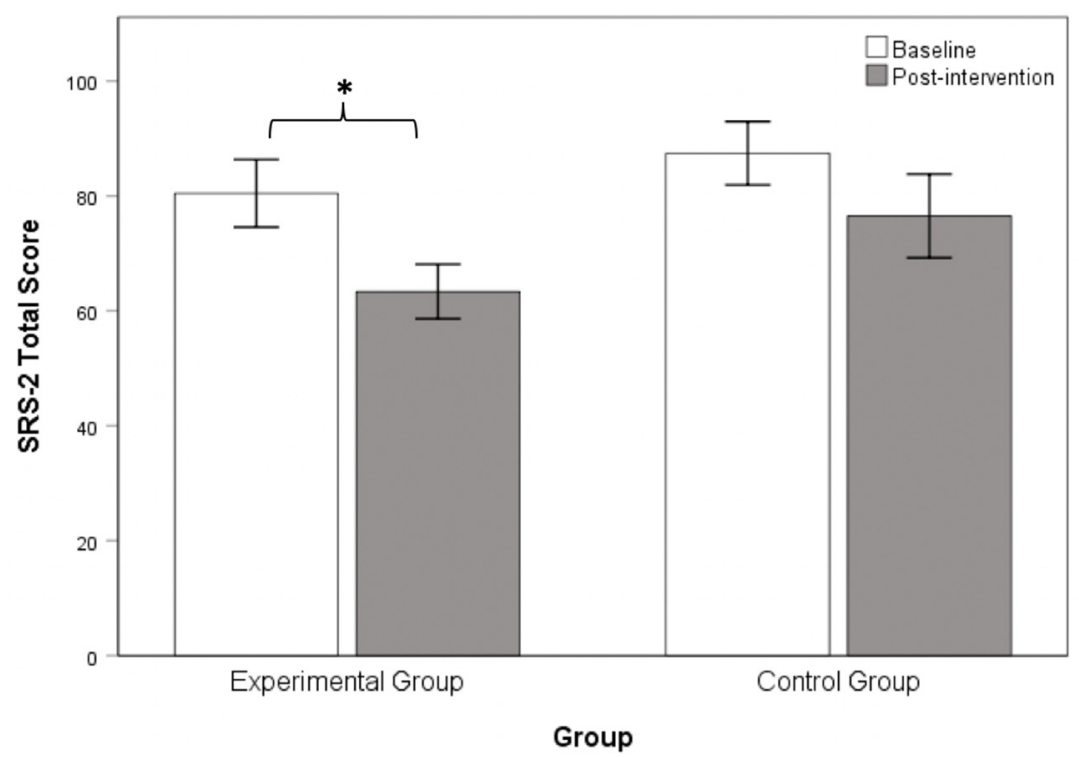

Figure 3. Mean parental rating on children's socialization ability in the experimental and control groups before and after intervention. Children from the experimental group are rated to have reduced social communication problems after intervention, whereas those in the control group were not. SRS-2 = Social Responsiveness Scale, 2nd edition (comprising measures of social communication ability and restricted and repetitive behavior). ${ }^{*} p<0.01$ in paired samples $t$-test. Error bars represent 1 standard error \pm mean.

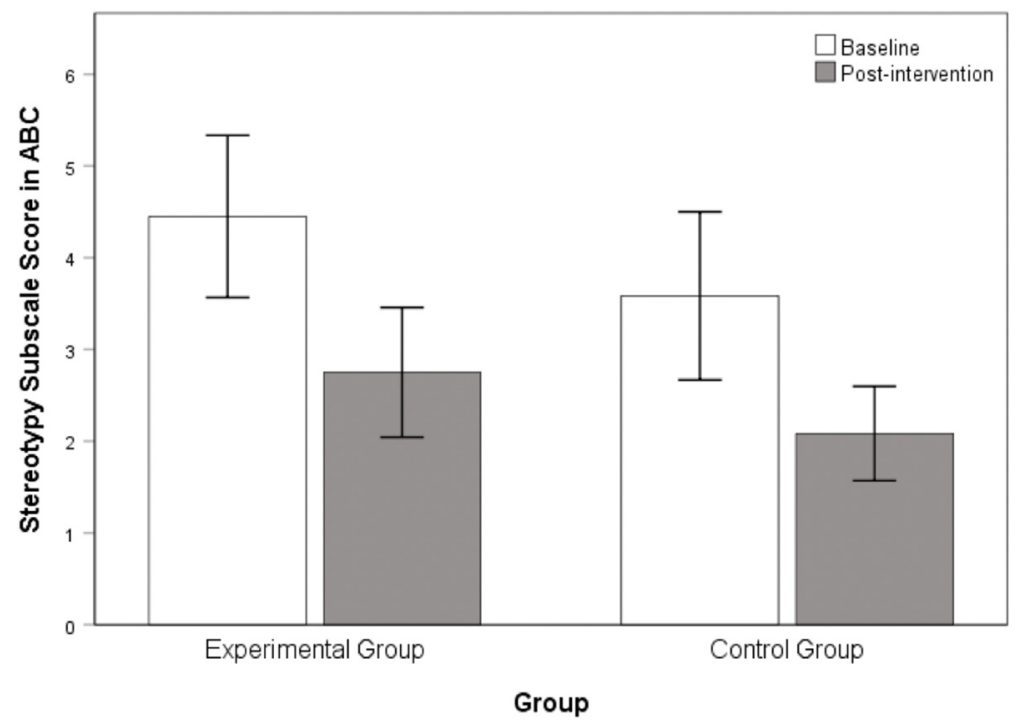

Figure 4. Mean parental rating on children's stereotypic/repetitive behaviors in the experimental and control group before and after intervention. Both groups show a reduced score in stereotypic behaviors. $\mathrm{ABC}=$ Aberrant Behavior Checklist. Error bars represent 1 standard error \pm mean. 

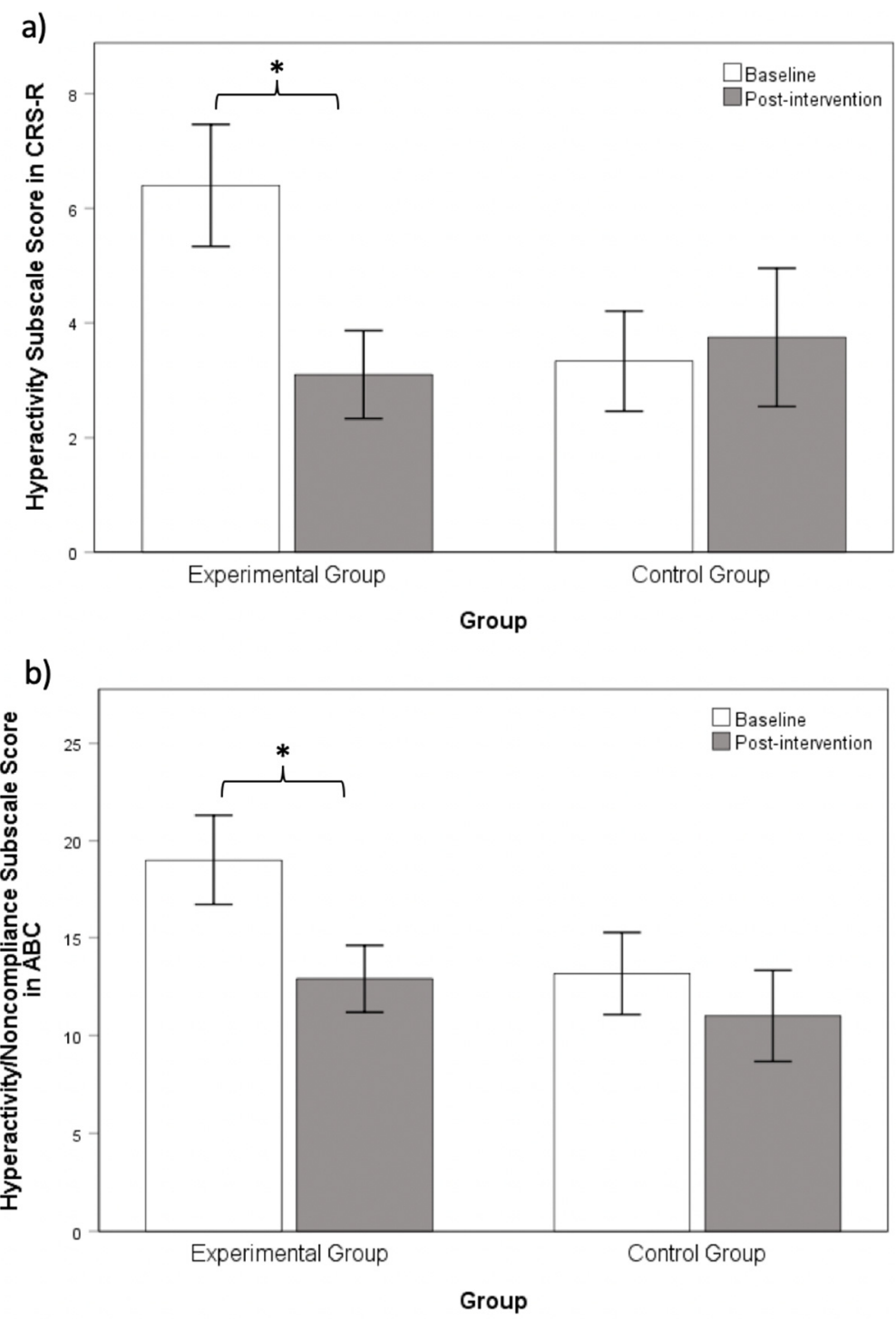

Figure 5. Mean parental rating on children's hyperactive behaviors in the experimental and control groups before and after intervention. The experimental group shows a significant reduction in hyperactivity in (a) CRS-R and (b) ABC after intervention, whereas the control group does not show such a reduction. CRS-R $=$ the short form of Conners' Rating Scales-Revised; $A B C=$ Aberrant Behavior Checklist. ${ }^{*} p<0.01$ in paired samples $t$-test. Error bars represent 1 standard error \pm mean.

\section{Discussion}

The present findings provide evidence for the potential benefits of the Chanwuyi Lifestyle Medicine Program on modulating immunological responses and behavioral problems in children with ASD. Children from the experimental group were found to have a significant downregulation of two pro-inflammatory chemokines, macrophages-related CCL2 and neutrophils-related CXCL8 and significantly reduced core autistic features (including social communication deficits and restricted/repetitive behavioral repertoire) and hyperactive behaviors after 7-month lifestyle changes. In contrast, children who were in the control group did not show such immunologic and behavioral changes. The present findings provide a potential immunologic-based explanation for our empirical and clinical evidence that many children with ASD would have improved cognitive functions and reduced allergic reactions (e.g., nasal, skin, and food allergy) after adopting the lifestyle changes according to the Chanwuyi principles [37-41].

CCL2 and CXCL8 are the most widely studied members of the CC and CXC chemokine families, respectively. The impact of deviated CCL2 and CXCL8 on neuroinflammation and 
pathogenesis of ASD has been implicated in past research. Xu et al. (2017) [52] suggested that CCL2 protein expression maximizes the probability of acute and chronic inflammation in the brain, which thereby affects an individual's level of cognitive functioning. Elevation of CCL2 and CXCL8 has been repeatedly reported to be linked with the inflammatory responses in the brain of patients with multiple sclerosis, stroke, traumatic brain injury, and Alzheimer's disease [53-56]. Increased CCL2 was also found to be associated with Parkinson's disease, encephalitis, and a brain tumor [57-59], whereas increased CXCL8 was found to be related to epilepsy, HIV-1, and neurosyphilis [60-62], The link between CCL2 and CXCL8 with ASD was also recurrently evidenced. Levels of chemokines macrophage chemoattractant CCL2 were found to be about two-fold higher in the anterior cingulate gyrus and cerebellum in the brain tissue of ASD than in the controls [4]. Moreover, cerebrospinal fluid from patients with autism showed a 12-fold increase in CCL2 when compared with controls [4]. Elevation of CCL2 in plasma was repeatedly reported in ASD [3,6,7]. Similarly, neutrophil chemotactic factor CXCL8 was found to be higher in the brain cortex of ASD in comparison to the control group [4]. Given the potent chemotactic effects of CXCL8 on neutrophils and granulocytes, Li et al. (2009) [5] have postulated that such elevation might implicate a chemokine-mediated neuroinflammation that could be related to possible brain damage led by infiltrated neutrophils and granulocytes. Furthermore, the increased peripheral level of CXCL8 in plasma samples was also found in children with ASD than typically developed children [3,6]. The increased CCL2 and CXCL8 have been reported to be associated with greater impairment in developmental and adaptive function and more frequent social withdrawal and stereotypic and hyperactive behaviors in ASD [6,7]. Therefore, the significant downregulation of CCL2 and CXCL8 in ASD after the 7-month Chanwuyi Lifestyle Medicine Program has shed some light on the potential benefits of the adopting a healthy lifestyle on improving the immunologic state of ASD.

As compared with CCL2 and CXCL8, the mechanism of CCL5 and CXCL9 implicated in the pathogenesis of ASD is relatively less studied. Although there was a marginal trend of reduction in CCL5 and increment in CXCL9 in the experimental group after the intervention, these changes are not statistically significant, which could be due to the large variability in their concentrations. Although allergy-related CCL5 has been found to be related to some neuroinflammatory diseases, such as encephalitis [58], multiple sclerosis [63], and Alzheimer's disease [64], it has a stronger linkage with HIV-related disease and cancers $[65,66]$. The association between CCL5 and ASD remains inconclusive. While a few studies have detected elevated CCL5 in ASD [3,7,10], other studies found a decline [67] or did not find an abnormal concentration of CCL5 in ASD [4]. Similarly, the role of Th1related CXCL9 in neuroinflammatory diseases has been minimally explored. Some studies have suggested its association with encephalitis, ischemic stroke, and epilepsy [68-70]. Few studies have addressed either an elevated or a suppressed level of CXCL9 in ASD [3,4]. Given the relatively less known mechanism of CCL5 and CXCL9 underlying pathogenesis of ASD, the non-significant treatment-induced alteration in these chemokines in the present study warrants further investigation to delineate the explanation for such results.

The therapeutic effect of Chanwuyi Lifestyle Medicine on changing the pro-inflammatory chemokine profile of ASD is theoretically sound. On the one hand, the Chan-based dietary recommendation of increasing intake of plant-based food and reducing intake of food that can generate excessive internal heat can reduce inflammatory responses in the body as well as the brain. This is in line with the repeated scientific findings of a plant-based diet on reducing the risk of having brain disorders associated with neuroinflammation (e.g., Alzheimer's disease [71], stroke [72]), and alleviating inflammatory responses in some physical illnesses (e.g., coronary heart disease, diabetes, and atherosclerosis [73]). A few studies have also suggested the modulating effect of diet change in altering immune responses in ASD [74]. On the other hand, the practice of a moderate intensity mind-body exercise (Neigong) and education on taking a more positive living attitude in the Chanwuyi Lifestyle Medicine Program are believed to enhance the capacity of children with ASD for promoting proper stress coping, which are protective factors for anti-neuroinflammation. The benefits of physical activity 
on reducing inflammation in autoimmune diseases such as diabetes and arthritis have been extensively studied with positive results [75]. A review conducted by Spielman, Little, and Klegeris [76] concluded that physical activity helped attenuate neuroinflammation associated with the major depressive disorder, schizophrenia, Alzheimer's disease, and Parkinson's disease. Positive life attitude, such as gratitude, can boost immune function and reduce the risk of stroke [77]. Although exercise-induced modulation of immune function in ASD has been rarely studied, children with ASD were found to be less physically active than typically developing children [78], and some research has supported the positive effects of physical activity (e.g., swimming, jogging, cycling, weight training, and walking) on improving their balance and flexibility [79], social communication skills [13], peer relations [79], and reducing stereotypical behaviors [15], inattention [79], and self-stimulation [79]. Therefore, it is worth exploring the potential benefits of regular exercise on the immunological function of ASD in the future.

The positive effects of the Chanwuyi Lifestyle Medicine Program on reducing autistic symptoms and impulse control problems (as reflected by hyperactivity) have been repeatedly demonstrated in our previous studies [37-39]. While our previous randomized controlled studies were focusing on the short-term effect (i.e., one month of training), the present study replicated similar positive outcomes over a longer term of intervention (i.e., 7 months). In the present study, the mean reduction of problematic behaviors in the experimental group (mean reduction: $35.8 \%$, range of changes: $21.3 \%$ to $51.6 \%$ ) is more than double that in the control group (mean reduction: $14.6 \%$, range of changes: $-12.6 \%$ to $41.9 \%$ ). As compared to the $13 \%$ reduction in problematic behaviors after one-month Neigong practice [39] and 21\% reduction after one-month Chan-based diet modification [38] reported in our previous studies, the extent of treatment effect $(35.8 \%)$ is relatively more substantial in the present study. The present greater effect could be related to the longer duration of intervention and the more comprehensive intervention protocol adopted. In previous studies, we have explored the neural mechanism underlying the treatment-induced behavioral changes and discovered enhanced brain activity in the anterior cingulate cortex in ASD [38,39]. Such findings imply that the Chanwuyi Lifestyle Medicine changes behaviors of ASD by altering their neural activity pattern. Given the association between behavioral deficits and immunological abnormality in ASD, the present study substantiated the positive effect of the Chanwuyi Lifestyle Medicine on the immunologic state of children with ASD, which could be a possible contributing factor for the positive behavioral changes in ASD.

Despite the encouraging findings in the present study, there are some limitations that warrant further studies. First, although both experimental and control groups are matched on age, intelligence level, immunological state, and behavioral symptoms, the large variability in some of the immunological measures could be related to the small sample size and/or varied age range. It is worth conducting a randomized controlled study with a larger sample size and a narrower range of age to examine the long-term effect of the Chanwuyi Lifestyle Medicine Program on the immunological function of ASD. Second, the participants in the present study are recruited based on convenience sampling, with those who are willing to receive the lifestyle intervention allocated to the experimental group, whereas those who are not willing or not possible to join the intervention group are allocated to the control group. Therefore, randomized controlled studies are needed to avoid potential bias of the present sampling technique and to verify the therapeutic effect of the Chanwuyi Lifestyle Medicine for ASD. Third, the Chanwuyi Lifestyle Medicine Program is developed upon the Chinese Chan-based medical model, which is probably relatively well-received by our participating children, who are Chinese. Whether such an intervention is also suitable and effective for people from Western societies warrants further investigation. Fourth, the present study has only revealed the alteration in plasma chemokines level and behavioral symptoms of ASD. Yet, the underlying neurological mechanism in relation to the changes in chemokines remains largely unknown. Therefore, to draw a conclusive remark on how the Chanwuyi Lifestyle Medicine Program influ- 
ences immunological function, which mediates alteration in brain physiological state and functioning and hence the behavioral symptoms in ASD, requires more extensive studies.

\section{Conclusions}

While effective intervention for modulating the immunological abnormalities of ASD remains largely unknown, the present study explores the potential therapeutic effects of a traditional Chinese lifestyle intervention, namely the Chanwuyi Lifestyle Medicine Program, on alleviating the deviation of chemokine concentration in children with ASD and on improving the common social and behavioral problems observed in ASD. We found that children with ASD having received a 7-month Chanwuyi Lifestyle Medicine Program showed significantly suppressed CCL2 and CXCL8 and a trend of reduced CCL5 and elevated CXCL9 in their peripheral blood samples, and they also had significantly reduced autistic symptoms and hyperactive behaviors. In contrast, children who did not receive the lifestyle intervention did not demonstrate such a pattern of changes. While previous studies on the Chanwuyi Lifestyle Medicine Program are mostly on its short-term effects (i.e., 1 month) on cognitive functions and problem behaviors, the present findings further substantiate the potential long-term (i.e., 7 months) benefits of such a lifestyle program on immune function and behaviors of ASD. However, further randomized controlled trials on the positive effects of the Chanwuyi Lifestyle Medicine Program on immune function are warranted, and it is worth exploring the immunological changes in relation with possible changes in brain functions and activity in future.

Author Contributions: Conceptualization, A.S.C.; methodology, A.S.C. and C.-k.W.; formal analysis, S.L.S., Y.M.Y.H. and I.M.T.C.; writing-original draft preparation, A.S.C., S.L.S. and Y.M.Y.H.; writing-review and editing, A.S.C., Y.M.Y.H., M.-c.C. and C.-k.W.; supervision, A.S.C.; project administration, S.L.S., Y.M.Y.H. and I.M.T.C.; funding acquisition, A.S.C. All authors have read and agreed to the published version of the manuscript.

Funding: This research was supported by a donation from S. H. Li and K. C. Shek to the Chinese University of Hong Kong.

Institutional Review Board Statement: The study was conducted according to the guidelines of the Declaration of Helsinki, and approved by the Ethical Review of Research Project Involving Human Subjects at the Hong Kong Polytechnic University (Reference No.: HSEARS20111215002).

Informed Consent Statement: Informed consent was obtained from parents of all participating children involved in the study.

Data Availability Statement: The data presented in this study are available on request from the corresponding author. The data are not publicly available due to privacy.

Acknowledgments: The authors would especially like to thank Venerable Master Dejian of the Songshan Monastery. The authors especially thank the registered nurses who helped draw blood and all children and teenagers and their parents who participated in the study. Thanks are also extended to Winnie Cheung, Debbie Yan, Lan He, Rex Wang, Carman Chu, Hannah Lee, and Thomas Lee, who assisted throughout the process of data collection, data management, and/or intervention.

Conflicts of Interest: The author is the founder of Chanwuyi Lifestyle Medicine Program. The funders had no role in the design of the study; in the collection, analyses, or interpretation of data, in the writing of the manuscript, or in the decision to publish the results.

\section{References}

1. Stubbs, E.G.; Crawford, M.L. Depressed lymphocyte responsiveness in autistic children. J. Autism Child. Schizophr. 1977, 7, 49-55. [CrossRef]

2. Young, A.M.H.; Chakrabarti, B.; Roberts, D.; Lai, M.C.; Suckling, J.; Baron-Cohen, S. From molecules to neural morphology: Understanding neuroinflammation in autism spectrum condition. Mol. Autism 2016, 7, 9. [CrossRef]

3. Han, Y.M.; Cheung, W.K.; Wong, C.K.; Sze, S.L.; Cheng, T.W.; Yeung, M.K.; Chan, A.S. Distinct cytokine and chemokine profiles in autism spectrum disorders. Front. Immunol. 2017, 8, 11. [CrossRef]

4. Vargas, D.L.; Nascimbene, C.; Krishnan, C.; Zimmerman, A.W.; Pardo, C.A. Neuroglial activation and neuroinflammation in the brain of patients with autism. Ann. Neurol. 2005, 57, 67-81. [CrossRef] [PubMed] 
5. Li, X.; Chauhan, A.; Sheikh, A.M.; Patil, S.; Chauhan, V.; Li, X.M.; Ji, L.; Brown, T.; Malik, M. Elevated immune response in the brain of autistic patients. J. Neuroimmunol. 2009, 207, 111-116. [CrossRef] [PubMed]

6. Ashwood, P.; Krakowiak, P.; Hertz-Picciotto, I.; Hansen, R.; Pessah, I.; Van de Water, J. Elevated plasma cytokines in autism spectrum disorders provide evidence of immune dysfunction and are associated with impaired behavioral outcome. Brain Behav. Immun. 2011, 25, 40-45. [CrossRef]

7. Ashwood, P.; Krakowiak, P.; Hertz-Picciotto, I.; Hansen, R.; Pessah, I.N.; Van de Water, J. Associations of impaired behaviors with elevated plasma chemokines in autism spectrum disorders. J. Neuroimmunol. 2011, 32, 196-199. [CrossRef] [PubMed]

8. Korzeniewski, S.J.; Allred, E.N.; O'Shea, T.M.; Leviton, A.; Kuban, K.C. Elevated protein concentrations in newborn blood and the risks of autism spectrum disorder, and of social impairment, at age 10 years among infants born before the 28th week of gestation. Transl. Psychiatry 2018, 8, 115. [CrossRef]

9. Leviton, A.; Joseph, R.M.; Allred, E.N.; Fichorova, R.N.; O'Shea, M.; Kuban, K.K.C.; Dammann, O. The risk of neurodevelopmental disorders at age 10 years associated with blood concentrations of interleukins 4 and 10 during the first postnatal month of children born extremely preterm. Cytokine 2018, 110, 181-188. [CrossRef]

10. Shen, Y.; Ou, J.; Liu, M.; Shi, L.; Li, Y.; Xiao, L.; Dong, H.; Zhang, F.; Xia, K.; Zhao, J. Altered plasma levels of chemokines in autism and their association with social behaviors. Psychiatry Res. 2016, 244, 300-305. [CrossRef]

11. Gupta, S. Immunological treatments for autism. J. Autism Dev. Disord. 2000, 30, 475-479. [CrossRef]

12. Gupta, S.; Samra, D.; Agrawal, S. Adaptive and innate immune responses in autism: Rationale for therapeutic use of intravenous immunoglobulin. J. Clin. Immunol. 2010, 30, 90-96. [CrossRef] [PubMed]

13. Pan, C.Y. Effects of water exercise swimming program on aquatic skills and social behaviors in children with autism spectrum disorder. Autism 2010, 14, 9-28. [CrossRef] [PubMed]

14. Elliott, R.O.; Dobbin, A.R.; Rose, G.D.; Soper, H.V. Vigorous, aerobic exercise versus general motor training activities: Effects on maladaptive and stereotypic behaviors of adults with both autism and mental retardation. J. Autism Dev. Disord. 1994, 24, 565-576. [CrossRef] [PubMed]

15. Yilmaz, I.; Yanardă̆, M.; Birkan, B.; Bumin, G. Effects of swimming training on physical fitness and water orientation in autism. Pediatr. Int. 2004, 46, 624-626. [CrossRef]

16. Powers, S.; Thibadeau, S.; Rose, K. Antecedent exercise and its effects on self-stimulation. Behav. Intervent. 1992, 7, 15-22. [CrossRef]

17. Ashwood, P.; Van de Water, J. A review of autism and the immune response. Clin. Dev. Immunol. 2004, 11, 165-174. [CrossRef] [PubMed]

18. Lucarelli, S.; Frediani, T.; Zingoni, A.M.; Ferruzzi, F.; Giardini, O.; Quintieri, F.; Barbato, M.; D’Eufemia, P.; Cardi, E. Food allergy and infantile autism. Panminerva Med. 1995, 37, 137-141. [PubMed]

19. American College of Lifestyle Medicine. Available online: https://lifestylemedicine.org (accessed on 21 November 2018).

20. Ornish, D.; Scherwitz, L.W.; Billings, J.H.; Gould, K.L.; Merritt, T.A.; Sparler, S.; Armstrong, W.T.; Ports, T.A.; Kirkeeide, R.L.; Hogeboom, C.; et al. Intensive lifestyle changes for reversal of coronary heart disease. JAMA 1998, 280, 2001-2007. [CrossRef]

21. Ornish, D.; Magbanua, M.J.M.; Weidner, G.; Weinberg, V.; Kemp, C.; Green, C.; Mattie, M.D.; Marlin, R.; Simko, J.; Shinohara, K.; et al. Changes in prostate gene expression in men undergoing an intensive nutrition and lifestyle intervention. Proc. Natl. Acad. Sci. USA 2008, 105, 8369-8374. [CrossRef]

22. Song, M.; Giovannucci, E. Preventable incidence and mortality of carcinoma associated with lifestyle factors among white adults in the United States. JAMA Oncol. 2016, 2, 1154-1161. [CrossRef] [PubMed]

23. Galimanis, A.; Mono, M.L.; Arnold, M.; Nedeltchev, K.; Mattle, H.P. Lifestyle and stroke risk: A review. Curr. Opin. Neurol. 2009, 22, 60-68. [CrossRef] [PubMed]

24. Gow, M.L.; Baur, L.A.; Johnson, N.A.; Cowell, C.T.; Garnett, S.P. Reversal of type 2 diabetes in youth who adhere to a very-lowenergy diet: A pilot study. Diabetologia 2017, 60, 406-415. [CrossRef]

25. Simon, A.U.; Collins, C.E.R. Lifestyle Redesign ${ }^{\circledR}$ for chronic pain management: A retrospective clinical efficacy study. Am. J. Occup. Ther. 2017, 71, 7104190040. [CrossRef]

26. Gardner, C.D.; Kiazand, A.; Alhassan, S.; Kim, S.; Stafford, R.S.; Balise, R.R.; Kraemer, H.C.; King, A.C. Comparison of the Atkins, Zone, Ornish, and LEARN diets for change in weight and related risk factors among overweight premenopausal women: The A TO Z Weight Loss Study: A randomized trial. JAMA 2007, 297, 969-977. [CrossRef]

27. Ornish, D.; Weidner, G.; Fair, W.R.; Marlin, R.; Pettengill, E.B.; Raisin, C.J.; Dunn-Emke, S.; Crutchfield, L.; Jacobs, F.N.; Barnard, R.J.; et al. Intensive lifestyle changes may affect the progression of prostate cancer. J. Urol. 2005, 174, 1065-1070. [CrossRef]

28. Chan, A.S. The Shaolin Chanwuyi: A Chinese Chan Buddhism; Chanwuyi Publishing: Hong Kong, China, 2010.

29. Chan, A.S. Contemporary Application of Shaolin Medicine: Dejian Mind- Body Intervention, 5th ed.; Chanwuyi Publishing: Hong Kong, China, 2013.

30. Chan, A.S.; Cheung, M.C.; Tsui, W.J.; Sze, S.L.; Shi, D. Dejian mind-body intervention on depressive mood of community-dwelling adults: A randomized controlled trial. Evid.-Based Complement. Altern. Med. 2011, 2011, 473961. [CrossRef]

31. Chan, A.S.; Cheung, M.C.; Sze, S.L.; Leung, W.W.M.; Shi, D. Shaolin Dan Tian breathing fosters relaxed and attentive mind: A randomized controlled neuro-electrophysiological study. Evid.-Based Complement. Altern. Med. 2011, 2011, 180704. [CrossRef]

32. Yu, R.; Woo, J.; Chan, A.S.; Sze, S.L. A Chinese Chan-based mind-body intervention improves psychological well-being and physical health of community-dwelling elderly: A pilot study. Clin. Interv. Aging 2014, 9, 727-736. [CrossRef] 
33. Chan, A.S.; Sze, S.L.; Woo, J.; Yu, R.H. A Chinese Chan-based lifestyle intervention improves memory of older adults. Front. Aging Neurosci. 2014, 6, 50. [CrossRef]

34. Chan, A.S.; Cheung, W.K.; Yeung, M.K.; Woo, J.; Kwok, T.; Shum, D.H.K.; Yu, R.; Cheung, M.C. A Chinese Chan-based Mind-Body Intervention Improves Memory of Older Adults. Front. Aging Neurosci. 2017, 9, 190. [CrossRef]

35. Chan, A.S.; Cheung, K.Y.; Yeung, M.K.; Lee, T.L. Sustained Effects of Memory and Lifestyle Interventions on Memory Functioning of Older Adults: An 18-Month Follow-Up Study. Front. Aging Neurosci. 2018, 10, 240. [CrossRef] [PubMed]

36. Chan, A.S.; Sze, S.L.; Dejian, S. Traditional Chinese Mind-Body Exercises Improve Self-Control Ability of an Adolescent with Asperger's Disorder. J. Psychol. Chin. Soc. 2008, 9, 225-239. [CrossRef]

37. Chan, A.S.; Sze, S.L.; Cheung, M.C.; Han, Y.M.; Leung, W.W.; Shi, D. Dejian mind-body intervention improves the cognitive functions of a child with autism. Evid.-Based Complement. Altern. Med. 2011, 2011, 549254. [CrossRef] [PubMed]

38. Chan, A.S.; Sze, S.L.; Han, Y.M.; Cheung, M.C. A Chan dietary intervention enhances executive functions and anterior cingulate activity in autism spectrum disorders: A randomized controlled trial. Evid.-Based Complement. Altern. Med. 2012, 2012, 262136. [CrossRef]

39. Chan, A.S.; Sze, S.L.; Siu, N.Y.; Lau, E.M.; Cheung, M.C. A Chinese mind-body exercise improves self-control of children with autism: A randomized controlled trial. PLoS ONE 2013, 8, e68184. [CrossRef]

40. Chan, A.S.Y.; Han, Y.M.Y.; Cheung, M.C. Chinese Chan-based prospective neuropsychological intervention for autistic children. In Comprehensive Guide to Autism; Patel, V.B., Preedy, V.R., Martin, C.R., Eds.; Springer: New York, NY, USA, 2014; pp. 2333-2355, ISBN 978-1-4614-4787-0.

41. Chan, A.S.; Han, Y.M.; Sze, S.L.; Lau, E.M. Neuroenhancement of memory for children with autism by a mind-body exercise. Front. Psychol. 2015, 6, 1893. [CrossRef] [PubMed]

42. Chan, A.S.; Wong, Q.Y.; Sze, S.L.; Kwong, P.P.; Han, Y.M.; Cheung, M.C. A Chinese Chan-based mind-body intervention for patients with depression. J. Affect. Disord. 2012, 142, 283-289. [CrossRef] [PubMed]

43. Chan, A.S.; Wong, Q.Y.; Sze, S.L.; Kwong, P.P.; Han, Y.M.; Cheung, M.C. A Chinese Chan-based mind-body intervention improves sleep on patients with depression: A randomized controlled trial. Sci. World J. 2012, 2012, 235206. [CrossRef]

44. Chan, A.S.; Han, Y.M.; Sze, S.L.; Wong, Q.Y.; Cheung, M.C. A randomized controlled neurophysiological study of a Chinese Chan-based mind-body intervention in patients with major depressive disorder. Evid.-Based Complement. Altern. Med. 2013, 2013, 812096. [CrossRef]

45. Chan, A.S.; Sze, S.L.; Cheung, M.C.; Lam, J.M.K.; Shi, D. Dejian mind-body intervention improves the functioning of a patient with chronic epilepsy: A case report. Cases J. 2009, 2, 9080. [CrossRef]

46. American Psychiatric Association. Diagnostic and Statistical Manual of Mental Disorders, 5th ed.; American Psychiatric Association: Arlington, VA, USA, 2013; ISBN 978-089-042-555-8.

47. Lord, C.; Rutter, M.; LeCouteur, A. Autism diagnostic interview-revised: A revised version of a diagnostic interview for caregivers of individuals with possible pervasive developmental disorders. J. Autism Dev. Disord. 1994, 24, 659-685. [CrossRef]

48. Wechsler, D. Wechsler Intelligence Scale for Children, 4th ed.; King-May Psychological Assessment: Hong Kong, China, 2010.

49. Constantino, J.N.; Gruber, C.P. The Social Responsiveness Scale Manual, 2nd ed.; Western Psychological Services: Los Angeles, CA, USA, 2012; ISBN 978-074-916-743-1.

50. Conners, C.K. Conners' Rating Scales-Revised: Short Form; Multi-Heath Systems: North Tonawanda, NY, USA, 1997.

51. Aman, M.G.; Singh, N.N. Aberrant Behavior Checklist: Manual; Slosson: East Aurora, NY, USA, 1986.

52. Xu, J.; Dong, H.; Qian, Q.; Zhang, X.; Wang, Y.; Jin, W.; Qian, Y. Astrocyte-derived CCL2 participates in surgery-induced cognitive dysfunction and neuroinflammation via evoking microglia activation. Behav. Brain Res. 2017, 332, 145-153. [CrossRef]

53. Bartosik-Psujek, H.; Stelmasiak, Z. The levels of chemokines CXCL8, CCL2 and CCL5 in multiple sclerosis patients are linked to the activity of the disease. Eur. J. Neurol. 2005, 12, 49-54. [CrossRef]

54. Semple, B.D.; Kossmann, T.; Morganti-Kossmann, M.C. Role of chemokines in CNS health and pathology: A focus on the CCL2/CCR2 and CXCL8/CXCR2 networks. J. Cereb. Blood Flow Metab. 2010, 30, 459-473. [CrossRef] [PubMed]

55. Corrêa, J.D.; Starling, D.; Teixeira, A.L.; Caramelli, P.; Silva, T.A. Chemokines in CSF of Alzheimer's disease patients. Arq. Neuropsiquiatr. 2011, 69, 455-459. [CrossRef]

56. Kimura, A.; Yoshikura, N.; Hayashi, Y.; Inuzuka, T. Cerebrospinal Fluid C-C Motif Chemokine Ligand 2 Correlates with Brain Atrophy and Cognitive Impairment in Alzheimer's Disease. J. Alzheimers Dis. 2018, 61, 581-588. [CrossRef] [PubMed]

57. Grozdanov, V.; Bliederhaeuser, C.; Ruf, W.P.; Roth, V.; Fundel-Clemens, K.; Zondler, L.; Brenner, D.; Martin-Villalba, A.; Hengerer, B.; Kassubek, J.; et al. Inflammatory dysregulation of blood monocytes in Parkinson's disease patients. Acta Neuropathol. 2014, 128, 651-663. [CrossRef]

58. Chowdhury, P.; Khan, S.A. Significance of CCL2, CCL5 and CCR2 polymorphisms for adverse prognosis of Japanese encephalitis from an endemic population of India. Sci. Rep. 2017, 7, 13716. [CrossRef] [PubMed]

59. Magge, S.N.; Malik, S.Z.; Royo, N.C.; Chen, H.I.; Yu, L.; Snyder, E.Y. Role of monocyte chemoattractant protein-1 (MCP-1/CCL2) in migration of neural progenitor cells toward glial tumors. J. Neurosci. 2009, 87, 1547-1555. [CrossRef]

60. Mazdeh, M.; Omrani, M.D.; Sayad, A.; Komaki, A.; Arsang-Jang, S.; Taheri, M.; Ghafouri-Fard, S. Expression analysis of cytokine coding genes in epileptic patients. Cytokine 2018, 110, 284-287. [CrossRef] 
61. Zheng, J.C.; Huang, Y.; Tang, K.; Cui, M.; Niemann, D.; Lopez, A.; Morgello, S.; Chen, S. HIV-1-infected and/or immune-activated macrophages regulate astrocyte CXCL8 production through IL-1 $\beta$ and TNF- $\alpha$ : Involvement of mitogen-activated protein kinases and protein kinase R. J. Neuroimmunol. 2008, 200, 100-110. [CrossRef]

62. Wang, C.; Wu, K.; Yu, Q.; Zhang, S.; Gao, Z.; Liu, Y.; Ni, L.; Cheng, Y.; Guan, Z.; Shi, M.; et al. CXCL13, CXCL10 and CXCL8 as potential biomarkers for the diagnosis of neurosyphilis patients. Sci. Rep. 2016, 6, 33569. [CrossRef] [PubMed]

63. Pittaluga, A. CCL5-Glutamate Cross-Talk in Astrocyte-Neuron Communication in Multiple Sclerosis. Front. Immunol. 2017, 8, 1079. [CrossRef]

64. Sankar, S.; Wood, L. Differences in inflammatory signaling between males and females with Alzheimer's disease. Alzheimers Dement. 2017, 13, 984. [CrossRef]

65. Aldinucci, D.; Colombatti, A. The inflammatory chemokine CCL5 and cancer progression. Mediat. Inflamm. 2014, 2014, 292376. [CrossRef]

66. Kelder, W.; McArthur, J.C.; Nance-Sproson, T.; McClernon, D.; Griffin, D.E. $\beta$-Chemokines MCP-1 and RANTES are selectively increased in cerebrospinal fluid of patients with human immunodeficiency virus-associated dementia. Ann. Neurol. 1998, 44, 831-835. [CrossRef] [PubMed]

67. Zerbo, O.; Yoshida, C.; Grether, J.K.; Van de Water, J.; Ashwood, P.; Delorenze, G.N.; Hansen, R.L.; Kharrazi, M.; Croen, L.A. Neonatal cytokines and chemokines and risk of autism spectrum disorder: The Early Markers for Autism (EMA) study: A case-control study. J. Neuroinflamm. 2014, 11, 113. [CrossRef] [PubMed]

68. Amin, M.; Vakilian, A.; Mahmoodi, M.H.; Hassanshahi, G.; Falahati-pour, S.K.; Dolatabadi, M.R.; Nadimi, A.E. Circulatory Levels of C-X-C Motif Chemokine Ligands 1, 9, and 10 Are Elevated in Patients with Ischemic Stroke. Eurasian J. Med. 2017, 49, 92-96. [CrossRef]

69. Lind, L.; Studahl, M.; Berg, L.P.; Eriksson, K. CXCL11 production in cerebrospinal fluid distinguishes herpes simplex meningitis from herpes simplex encephalitis. J. Immunol. 2017, 14, 134. [CrossRef] [PubMed]

70. Farias-Moeller, R.; LaFrance-Corey, R.; Bartolini, L.; Wells, E.M.; Baker, M.; Doslea, A.; Suslovic, W.; Greenberg, J.; Carpenter, J.L.; Howe, C.L. Fueling the FIRES: Hemophagocytic lymphohistiocytosis in febrile infection-related epilepsy syndrome. Epilepsia 2018, 59, 1753-1763. [CrossRef]

71. Morris, M.C.; Tangney, C.C.; Wang, Y.; Sacks, F.M.; Bennett, D.A.; Aggarwal, N.T. MIND diet associated with reduced incidence of Alzheimer's disease. Alzheimers Dement. 2015, 11, 1007-1014. [CrossRef] [PubMed]

72. Campbell, T. A plant-based diet and stroke. J. Geriatr. Cardiol. 2017, 14, 321-326. [CrossRef]

73. O'Keefe, J.H.; Gheewala, N.M.; O'Keefe, J.O. Dietary strategies for improving post-prandial glucose, lipids, inflammation, and cardiovascular health. J. Am. Coll. Cardiol. 2018, 51, 249-255. [CrossRef]

74. Jyonouchi, H.; Geng, L.; Ruby, A.; Zimmerman-Bier, B. Dysregulated innate immune responses in young children with autism spectrum disorders: Their relationship to gastrointestinal symptoms and dietary intervention. Neuropsychobiology 2005, 51, 77-85. [CrossRef] [PubMed]

75. Gleeson, M.; Bishop, N.C.; Stensel, D.J.; Lindley, M.R.; Mastana, S.S.; Nimmo, M.A. The anti-inflammatory effects of exercise: Mechanisms and implications for the prevention and treatment of disease. Nat. Rev. Immunol. 2011, 11, 607-615. [CrossRef]

76. Spielman, L.J.; Little, J.P.; Klegeris, A. Physical activity and exercise attenuate neuroinflammation in neurological diseases. Brain Res. Bull. 2016, 125, 19-29. [CrossRef] [PubMed]

77. Emmons, R.A.; Stern, R. Gratitude as a psychotherapeutic intervention. J. Clin. Psychol. 2013, 69, 846-855. [CrossRef]

78. Pan, C.Y. Objectively measured physical activity between children with autism spectrum disorders and children without disabilities during inclusive recess settings in Taiwan. J. Autism Dev. Disord. 2008, 38, 1292-1301. [CrossRef] [PubMed]

79. Sowa, M.; Meulenbroek, R. Effects of physical exercise on autism spectrum disorders: A meta-analysis. Res. Autism Spectr. Disord. 2012, 6, 46-57. [CrossRef] 